\title{
The big misunderstanding in rheumatology: Ultrasound is just equipment it cannot replace a doctor!
}

\author{
Georgios Filippou ${ }^{1}$, Annamaria Iagnocco ${ }^{2}$
}

${ }^{1}$ Department of Medicine, Surgery and Neuroscience, Rheumatology Department, University of Siena, Siena, ${ }^{2}$ Ultrasound Unit, Dipartimento Medicina Interna e Specialità Mediche, Sapienza Università di Roma, Rome, Italy

Most of the articles, including ours, dealing with any application of ultrasound (US) in rheumatic diseases, start with a phrase that sounds like: "in recent years, US has gained a central role as an important tool for Rheumatologist clinical practice". However, this is probably only the "sonographers" perception as currently US is not included in the diagnostic classification/criteria for the most important rheumatic diseases and also in many cases it is not recommended as the principal tool for the assessment and follow-up of patients. This is particularly relevant regarding rheumatoid arthritis (RA).

Some recently published reviews [1] and the EULAR recommendations on the use of imaging for the clinical management of RA [2] have highlighted that current evidence for the correct use of US for the monitoring of patients with RA is not strong enough to allow the definition of any recommendation on the use of US as a principal tool for this task. The point is that US has not clearly and unequivocally offered, in the very few available studies, any advantage over the use of clinical indices alone for this purpose. But how is this possible?

It is common knowledge and it has been very well demonstrated, that US is capable of revealing more synovitis than a clinical examination in joints of patients affected by RA as also acknowledged by EULAR experts [2]. If this is true and if synovitis is the reason for joint damage progression, should US not be the most appropriate exam to predict joint damage? One of the papers

Received Accepted

Med Ultrason

2016, Vol. 18, No 3, 273-274

Corresponding author: Annamaria Iagnocco, Dipartimento Medicina Interna e Specialità Mediche: Reumatologia, Sapienza Università di Roma,

V.le del Policlinico 155, Rome - 00161, Italy.

Phone: +3906 49974634, Fax: +39 0649974642

E-mail: annamaria.iagnocco@uniroma1.it that have tried to address the utility of US and clinical synovitis in predicting joint damage progression over a two year period was published in 2012 by Dougados et al [3]. In that study, 59 patients were assessed with US and clinical examination for synovitis at baseline and after a 4 month period and by conventional radiology at baseline and after 2 years for the evaluation of joint damage progression. The mean age of patients was 59 years old $( \pm 12)$, prevalently overweight (BMI $25 \pm 5 \mathrm{~kg} /$ $\left.\mathrm{m}^{2}\right)$, females $(81 \%)$, with a long standing disease $(10 \pm 8$ years) and a relatively high percentage of surgery for RA ( $24 \%$ of patients). In this cohort of patients, joint damage progression could be due to several reasons, for example biomechanical modifications or the overloading of healthy joints, and the evaluation of joint space narrowing as an outcome could be misleading regarding the ability of synovitis to predict joint damage independently of the method used to assess it.

This is just an example of how difficult it could be to design a study that would address the fateful question: is US better than a clinical examination for patient follow up? The perfect study should be carried out in young patients without other comorbidities, who do similar jobs (similar stress to the joints), who have no joint damage at the baseline, who are administered exactly the same treatment, who can be followed up for a sufficiently long period (years), who are always assessed by the same assessors for the whole period in a blind manner and many other aspects that are practically impossible to carry out in a real life study. This means that it would be really difficult to address this issue. But do we really need all this evidence? Maybe we already have it!

Hippocrates, the father of Western Medicine, the one we currently practice, based his science on observations. He was the first to use a rectal speculum, an "endoscopic" approach in the history of medicine and he was also the first thoracic surgeon to use lead pipes to drain chest 
wall abscesses. He treated many patients and he spent his life observing and treating patients with the aim of categorising illnesses (acute, chronic, endemic and epidemic) and understanding their course coining phrases

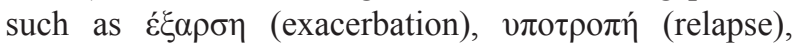

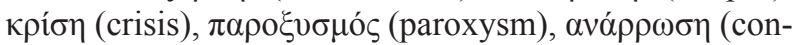
valescence) and others still in use in modern medicine. In other words, since ancient times, doctors and researchers have been just trying to obtain one thing: information! And they were disposed to do their best in order to get it.

In the $21^{\text {st }}$ century, we practise the exact opposite phenomenon. We are able to obtain such relevant information and in such an easy way but we are not ready to handle it! This is the case of US in rheumatology. With a simple, quick and harmless exam we can obtain all the necessary information and even more data on the status of the joint, tendons, periarticular tissues of patients with RA. We can make an accurate differential diagnosis, identifying with disarming precision, the site and the entity of the pathology and also understand if a treatment works or not... by simply placing a probe on a joint.

The rheumatologic scientific community is currently discussing whether US adds any advantages or not for the follow up of patients with RA with respect to clinical examination alone. However, this is not the correct question to ask. The relevant point is: are all rheumatologists ready to use and handle all the information that US can provide? There is no answer to this question. What is crystal clear is that a clinical examination and US "see" different aspects of the same disease [4]. Maybe, if we really want to make some progress in this field, new studies should not be addressed to identify if US is more useful than clinimetric indices in RA, but only to understand what is or is not important from the enormous amount of information that US (and imaging generally) provides to the clinician. US captures many different aspects and probably not all of them have the same importance in a given moment of the disease in all patients. This is the real challenge for the future. To compare US to a clinical examination is like comparing a supercar to an old city car. Both of them can take you to your destination, but if you can correctly handle the supercar you will arrive quicker and safer; if not, you would be better using the old city car in order to avoid any potential accidents.

It is clear that in modern medicine it is impossible to be an "expert" in many fields and from our point of view there is only one solution to this problem. Those of us who are willing to train, study and understand US should go ahead and continue or start to assess our patients using US. Those of us who are not, would be better continuing to use the old methods. US is just equipment; we should not expect it to take the place of the decision maker: the doctor!

\section{References}

1. Mandl P, Kurucz R, Niedermayer D, Balint PV, Smolen JS. Contributions of ultrasound beyond clinical data in assessing inflammatory disease activity in rheumatoid arthritis: current insights and future prospects. Rheumatology 2014; 53: 2136-2142.

2. Colebatch AN, Edwards CJ, Ostergaard M, et al. EULAR recommendations for the use of imaging of the joints in the clinical management of rheumatoid arthritis. Ann Rheum Dis 2013; 72: 804-814.

3. Dougados M, Devauchelle-Pensec V, Ferlet JF, et al. The ability of synovitis to predict structural damage in rheumatoid arthritis: a comparative study between clinical examination and ultrasound. Ann Rheum Dis 2013; 72: 665-671.

4. D'Agostino M-A, Boers M, Wakefield RJ, et al. Exploring a new ultrasound score as a clinical predictive tool in patients with rheumatoid arthritis starting abatacept: results from the APPRAISE study. RMD Open 2016; 2: e000237. 